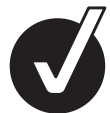

EDITOR'S

CHOICE

\title{
Metabolic surgery profoundly influences gut microbial-host metabolic cross-talk
}

Jia V Li, ${ }^{1,2}$ Hutan Ashrafian,, ${ }^{2,3}$ Marco Bueter, ${ }^{3,4}$ James Kinross, ${ }^{1,2}$ Caroline Sands, ${ }^{1}$ Carel W le Roux, ${ }^{3}$ Stephen R Bloom, ${ }^{3}$ Ara Darzi, ${ }^{2}$ Thanos Athanasiou, ${ }^{2}$ Julian R Marchesi, ${ }^{5}$ Jeremy K Nicholson, ${ }^{1,2}$ Elaine Holmes ${ }^{1}$

\section{See Commentary, p 1166}

- Additional tables and figures are published online only. To view these files, please visit the journal online (http://gut.bmj. com)

${ }^{1}$ Section of Biomolecular Medicine, Imperial College London, London, UK

${ }^{2}$ Section of Biosurgery \& Surgical Technology, Department of Surgery and Cancer, Imperial College London, London, UK

${ }^{3}$ Section of Investigative Medicine, Division of Diabetes, Endocrinology \& Metabolism, Department of Medicine, Faculty of Medicine, Imperial College London, London, UK ${ }^{4}$ Department of Surgery, Division of Visceral and Transplantation Surgery, University Hospital, Zürich, Switzerland

${ }^{5}$ School of Biosciences, Cardiff University, Cardiff, UK

\section{Correspondence to}

Professor Elaine Holmes, Department of Surgery and Cancer, Sir Alexander Fleming Building, Imperial College London, South Kensington, London SW7 2AZ, UK: elaine.holmes@imperial.ac.uk Dr Julian R Marchesi, School of Biosciences, Cardiff University, Museum Avenue, Cardiff, CF10 3AT, UK;

marchesiji@cardiff.ac.uk

JVL and HA contributed equally to this work.

Revised 16 March 2011 Accepted 17 March 2011 Published Online First 14 May 2011

\begin{abstract}
Background and aims Bariatric surgery is increasingly performed worldwide to treat morbid obesity and is also known as metabolic surgery to reflect its beneficial metabolic effects especially with respect to improvement in type 2 diabetes. Understanding surgical weight loss mechanisms and metabolic modulation is required to enhance patient benefits and operative outcomes.
\end{abstract}

Methods The authors applied a parallel and statistically integrated bacterial profiling and metabonomic approach to characterise Roux-en-Y gastric bypass (RYGB) effects in a non-obese rat model.

Results Substantial shifts of the main gut phyla towards higher concentrations of Proteobacteria (52-fold), specifically Enterobacter hormaechei, are shown. Low concentrations of Firmicutes (4.5-fold) and Bacteroidetes (twofold) in comparison with sham-operated rats were also found. Faecal extraction studies revealed a decrease in faecal bile acids and a shift from protein degradation to putrefaction through decreased faecal tyrosine with concomitant increases in faecal putrescine and diaminoethane. Decreased urinary amines and cresols were found and indices of modulated energy metabolism were demonstrated after RYGB, including decreased urinary succinate, 2-oxoglutarate, citrate and fumarate. These changes could also indicate renal tubular acidosis, which is associated with increased flux of mitochondrial tricarboxylic acid cycle intermediates. A surgically induced effect on the gut-brain-liver metabolic axis is inferred from modulated faecal $\gamma$-aminobutyric acid and glutamate.

Conclusion This profound co-dependence of mammalian and microbial metabolism, which is systematically altered after RYGB surgery, suggests that RYGB exerts local and global metabolic effects. The effect of RYGB surgery on the host metabolic-microbial cross-talk augments our understanding of the metabolic phenotype of bariatric procedures and can facilitate enhanced treatments for obesity-related diseases.

\section{INTRODUCTION}

Obesity is a global healthcare epidemic that affects all ages and socioeconomic groups. The World Health Organization ${ }^{1}$ projects a rising prevalence of obesity by approximately seven million per year, associated with a concomitant rise in obesityrelated co-morbidities such as diabetes, ${ }^{2}$ metabolic syndrome, heart disease, ${ }^{3} 4$ sleep apnoea ${ }^{5}$ and cancer. ${ }^{6}$ These conditions lead to significant morbidity and mortality, which result in increased healthcare costs and consumption of medical

\section{Significance of this study}

What is already known about this subject?

- Bariatric surgery, also-called metabolic surgery, is an effective approach to treating morbid obesity and can achieve pronounced metabolic effects including the resolution of type 2 diabetes.

- The Roux-en-Y gastric bypass (RYGB) operation is a bariatric procedure that achieves its physiological benefits through the BRAVE effects: Bile flow alteration, Reduction of gastric size, Anatomical gut rearrangement and altered flow of nutrients, Vagal manipulation, and subsequent Enteric gut hormone modulation.

- Recent studies have shown the effect of bariatric bypass on the microbial composition of obese people, including an increase in Gammaproteobacteria and a decrease in Firmicutes after RYGB surgery.

- Some metabolic profiling studies have identified the altered concentrations of serum biochemical components such as $p$-cresyl sulphate, nervonic acid and lysophosphatidylcholine after RYGB surgery.

What are the new findings?

- The RYGB operation induces substantial shifts of the main gut phyla towards higher concentrations of Proteobacteria (52-fold), specifically Enterobacter hormaechei, and lower concentrations of Firmicutes (4.5-fold) and Bacteroidetes (twofold) in comparison with sham-operated rats.

- The surgically altered anatomy and flow of nutrients is associated with a global change in urinary and faecal profiles reflecting (a) increased activity of oligosaccharide fermentation in the gut, (b) biogenesis of $p$-cresol and related compounds, (c) generation of amines, and (d) lower concentrations of urinary tricarboxylic acid intermediates, which may mechanistically contribute to body weight loss and metabolic enhancements.

- Faecal profiles reveal a decrease in faecal bile acids and a shift from protein degradation to putrefaction through decreased faecal tyrosine with concomitant increases in faecal putrescine and diaminoethane.

How might it impact on clinical practice in the foreseeable future?

- Understanding host-microbial cross-talk and the metabolic phenotype of bariatric procedures should facilitate management strategies for obesity-related disease. This should lead to improved operative procedures and novel treatments for obesity and metabolic disease. 
resources. ${ }^{6}$ Although a number of treatment strategies have been applied to obesity, including lifestyle changes, behavioural therapy and pharmacotherapies, the results have only been marginally beneficial for morbidly obese patients. ${ }^{7}$

Bariatric surgical approaches to reducing body fat have provided a definitive treatment for morbid obesity. These operations are successful in achieving and maintaining long-term weight loss, ${ }^{8}$ but also achieve pronounced metabolic effects, including the resolution of type 2 diabetes in up to $75 \%$ of patients through both weight-dependent and weight-independent activities. ${ }^{39}$ As a result, these procedures are now considered to be 'metabolic operations', achieving a significant beneficial effect on metabolism and physiology. ${ }^{10-12}$

The mechanisms of weight loss and metabolic augmentation through metabolic surgery remain poorly understood. Roux-en-Y gastric bypass (RYGB) is a multimodal surgical procedure that consists of Bile flow alteration, Reduction of gastric size, Anatomical gut rearrangement and altered flow of nutrients, Vagal manipulation, and subsequent Enteric gut hormone modulation - the BRAVE effects. ${ }^{4-11}$ Although modulation of gut hormones has been shown to contribute to the beneficial effects of surgery (primarily those of the hindgut such as the L-cell-secreted glucagon-like peptide-1 (GLP-1) and peptide YY (PYY) to modify diabetes and appetite), ${ }^{9} 12$ they do not account for all the metabolic changes associated with these operations. Elucidating the beneficial mechanisms of metabolic surgery should (a) help identify patients most likely to have a successful bariatric surgical outcome through the application of pharmacometabonomic approaches ${ }^{13}$ and $(b)$ identify biochemical mechanisms that may give new druggable antidiabetic and obesity targets.

A practical method for studying the global metabolic effects of surgery is through top-down systems biology and metabolic phenotyping approaches, which, if applied at individual and population levels, could lead to advances in both personalised healthcare, such as stratified chemotherapeutic decision-making, and molecular epidemiology, including risk biomarkers and risk hypothesis evaluation. ${ }^{14}$ This approach has been used extensively to study metabolic syndrome, cancer, infectious diseases and nutritional interventions. ${ }^{15} 16$ Many of the metabolic profiling studies of animal models of obesity to date have implicated a strong influence of gut microbial metabolism through altered urinary co-metabolism including urinary phenols, bile acids and methylamines. ${ }^{17} 18$ Parallel studies using microbial data have also shown that obese and lean people have profound differences in their gut microbial landscape, with a major shift towards an increased Firmicutes to Bacteroidetes ratio ${ }^{19}$ in obese individuals (which is responsive to a low-calorie diet). ${ }^{20}$ However, Duncan et $a l^{21}$ have demonstrated that this ratio is not associated with obesity in all human studies. An increase in gut Actinobacteria in obese people has also been observed in a core gut microbiome study of obese and lean twins. ${ }^{20}$ The study by Zhang et al further investigated the effect of bariatric bypass on three obese patients and demonstrated an increase in Gammaproteobacteria and a decrease in Firmicutes after surgery compared with three normal-weight controls and three obese patients. ${ }^{22}$ Another recent study on 30 obese individuals and 13 lean control human subjects has shown higher concentrations of Escherichia coli species 3 months after the RYGB operation, which was inversely associated with body fat mass, and reported that Faecalibacterium prausnitzii species correlate with reduced low-grade inflammation in obesity and diabetes. ${ }^{23}$ A separate study in 14 female patients exploring the metabolic effects of bariatric surgery showed an increase in serum concentrations of $p$-cresyl sulphate, nervonic acid and lysophosphatidylcholine 6 months after surgery. ${ }^{24}$ These metabolic changes may therefore represent the effects of metabolic surgery on renal function, insulin sensitivity and lipid metabolism, respectively.

Since both mammalian and gut microbial metabolism exhibit interdependence with respect to controlling energy balance, we used a parallel microbial and metabolic profiling strategy based on 454 pyrosequencing technology and ${ }^{1} \mathrm{H}$ nuclear magnetic resonance (NMR) spectroscopy, respectively, to explore the impact of RYGB surgery on interactions between the faecal and urinary metabolic phenotype, faecal bile acids and gut microbial modulation in non-obese Wistar rats.

\section{MATERIALS AND METHODS Animal model and sampling}

Male Wistar rats (non-obese) were individually housed under a $12 \mathrm{~h} / 12 \mathrm{~h}$ light/dark cycle at a room temperature of $21 \pm 2^{\circ} \mathrm{C}$. Water and standard chow were available ad libitum, unless otherwise stated. All experiments were performed under a licence issued by the UK Home Office (PL 70-6669). Rats were acclimatised for 1 week and randomised to RYGB or sham operation according to our previously described technique. ${ }^{10}$

Rats were food deprived overnight for $12 \mathrm{~h}$ before the operation with water available ad libitum. They were weighed and then anaesthetised with isofluorane (4\% for induction, 3\% for maintenance). Preoperative antibacterial prophylaxis was administered intraperitoneally to both RYGB and sham groups ( $1 \mathrm{ml}$ amoxicillin/flucoxacillin solution, both at $12.5 \mathrm{mg} / \mathrm{ml}$ ). Surgery was performed on a heating pad to avoid any decrease in body temperature during the procedure. The abdomen was shaved and disinfected with surgical scrub and a midline laparotomy was performed.

The sham procedure consisted of a $7 \mathrm{~mm}$ gastrotomy on the anterior wall of the stomach with subsequent closure (interrupted prolene 5-0 sutures) and a $7 \mathrm{~mm}$ jejunotomy with subsequent closure (running prolene 6-0 suture). In the gastric bypass procedure, the proximal jejunum was divided $15 \mathrm{~cm}$ distal to the pylorus to create a biliopancreatic limb. After identification of the caecum, the ileum was followed proximally to create a common channel of $25 \mathrm{~cm}$. Here, a $7 \mathrm{~mm}$ side-to-side jejunojejunostomy (running prolene 7-0 suture) between the biliopancreatic limb and the common channel was performed. The gastric pouch and alimentary limb were anastomosed endto-side using a running prolene 7-0 suture. The gastric remnant was closed with interrupted prolene 5-0 sutures. The complete bypass procedure lasted $\sim 60 \mathrm{~min}$, and the abdominal wall was closed in layers using 4-0 and 5-0 prolene sutures. Approximately $20 \mathrm{~min}$ before the anticipated end of general anaesthesia, all rats were injected with $0.1 \mathrm{ml} 0.3 \%$ buprenorphine subcutaneously to minimise postoperative discomfort. Immediately after abdominal closure, all rats were injected subcutaneously with $5 \mathrm{ml}$ normal saline to compensate for intraoperative fluid loss. After $24 \mathrm{~h}$ of wet diet (normal chow soaked in tap water), regular chow was offered on postoperative day 2 .

Urine and stool were collected for $24 \mathrm{~h}$ at 2 weeks, 4 weeks, 6 weeks and 8 weeks after the operation and stored at $-80^{\circ} \mathrm{C}$.

\section{NMR spectroscopy}

Urine samples were thoroughly defrosted and vortex-mixed for $15 \mathrm{~s}$. An aliquot of $400 \mu \mathrm{l}$ was then mixed with $250 \mu \mathrm{l} 0.2 \mathrm{M}$ sodium phosphate buffer ( $\mathrm{pH}=7.4$ ) containing $20 \%$ deuterium oxide $\left(\mathrm{D}_{2} \mathrm{O}\right)$ for the magnetic field lock, $0.01 \% 3$-(trimethylsilyl)$\left[2,2,3,3-{ }^{2} \mathrm{H}_{4}\right]$ propionic acid sodium salt (TSP) for the spectral 
calibration, and $3 \mathrm{mM}$ sodium azide $\left(\mathrm{Na}_{3} \mathrm{~N}\right)$ to avoid bacterial contamination. The resulting mixture was centrifuged at $10392 \mathrm{~g}$ for $10 \mathrm{~min}$, and $600 \mu \mathrm{l}$ supernatant was transferred to a NMR tube with an outer diameter of $5 \mathrm{~mm}$ pending ${ }^{1} \mathrm{H}$ NMR spectral acquisition.

One faecal pellet was placed into a $2 \mathrm{ml}$ Eppendorf tube containing $1.4 \mathrm{ml}$ of the phosphate buffer used for urinary sample preparation. The sample was homogenised, vortexmixed for $15 \mathrm{~s}$, sonicated for $30 \mathrm{~min}$ at $298 \mathrm{~K}$, and centrifuged at $10392 \mathrm{~g}$ for $20 \mathrm{~min}$. A $700 \mu \mathrm{l}$ volume of supernatant was placed in a $1.5 \mathrm{ml}$ Eppendorf tube and centrifuged again under the same conditions. The supernatant $(600 \mu \mathrm{l})$ was put in a NMR tube with an outer diameter of $5 \mathrm{~mm}$ pending ${ }^{1} \mathrm{H}$ NMR spectral acquisition.

${ }^{1} \mathrm{H}$ NMR spectra of urine and faecal extract samples were obtained using a Bruker $600 \mathrm{MHz}$ spectrometer (Bruker, Rheinstetten, Germany) at the operating ${ }^{1} \mathrm{H}$ frequency of $600.13 \mathrm{MHz}$ at a temperature of $300 \mathrm{~K}$. A standard NMR pulse sequence (recycle delay $-90^{\circ}-\mathrm{t}_{1}-90^{\circ}-\mathrm{t}_{\mathrm{m}}-90^{\circ}$-acquisition) was applied to acquire one-dimensional ${ }^{1} \mathrm{H}$ NMR spectral data, where $t_{1}$ was set to $3 \mu$ s and $t_{m}$ (mixing time) was set to $100 \mathrm{~ms}$. The water peak suppression was achieved using selective irradiation during a recycle delay of $2 \mathrm{~s}$ and $\mathrm{t}_{\mathrm{m}}$. A $90^{\circ}$ pulse was adjusted to $\sim 10 \mu \mathrm{s}$. A total of 128 scans were collected into $64 \mathrm{k}$ data points with a spectral width of $20 \mathrm{ppm}$. A series of two-dimensional NMR spectra including ${ }^{1} \mathrm{H}-{ }^{1} \mathrm{H}$ correlation spectroscopy, ${ }^{1} \mathrm{H}-{ }^{1} \mathrm{H}$ total correlation spectroscopy, J-resolved spectroscopy, ${ }^{1} \mathrm{H}-{ }^{13} \mathrm{C}$ heteronuclear single-quantum coherence and ${ }^{1} \mathrm{H}-{ }^{13} \mathrm{C}$ heteronuclear multiple-bond coherence were acquired on selected urine and faecal extract samples for the purpose of metabolite annotations. The standard parameters used for these spectral acquisitions have previously been reported. ${ }^{25} 26$

\section{Multivariate data analysis}

Multivariate data analyses were performed on the basis of the prepossessed NMR dataset. ${ }^{1} \mathrm{H}$ NMR spectra obtained from urine and faecal extracts were automatically phased, referenced to TSP at $\beta 0.00$ and baseline-corrected using a MATLAB script developed by $\mathrm{Dr} T$ Ebbels at Imperial College. The resulting NMR spectra $(\delta 0-10)$ were imported to MATLAB software and digitised into $20 \mathrm{k}$ data points with the resolution of 0.0005 using script developed in-house ( $\mathrm{Dr} O \mathrm{Cloarec}$ ). The water peak region $84.62-5.05$ in urine spectra and $84.7-4.9$ in faecal water spectra were removed to minimise the effect of the disordered baseline. In addition, regions $\delta 0-0.10, \delta 5.47-6.24$ and $\delta 9.90-10.00$ in urine spectra, and regions $\delta 0-0.30$ and \$9.4-10.00 in faecal water spectra containing only noise were therefore removed, and this was followed by normalisation to the remaining spectral areas of NMR data in order to perform further analyses. Principal component analysis, O-PLS and OPLS-DA were carried out on the basis of the resulting NMR spectral datasets in SIMCA $(P+11.5)$ and MATLAB (2009a) software.

\section{Analysis of the gut microbiota by pyrosequencing}

The composition of the gut microbiota was determined by surveying the $16 \mathrm{~S}$ rRNA genes in each animal. DNA was extracted from faecal pellets $(250 \mathrm{mg}$ ) using a modified protocol based on the Qiagen Stool Kit (Qiagen, Crawly, UK) with an additional bead-beating step to homogenise and lyse bacteria in the samples $(0.1 \mathrm{~g} 0.1 \mathrm{~mm}$ sterile glass beads, $3 \times 4500 \mathrm{rpm}$ for $30 \mathrm{~s}$ with $5 \mathrm{~min}$ on ice between cycles). DNA obtained from this extraction was quantified using the Invitrogen Qubit platform and diluted to a working concentration of $10 \mathrm{ng} / \mu \mathrm{l}$. PCR was used to amplify the $\mathrm{V} 1-\mathrm{V} 3$ regions of the $16 \mathrm{~S}$ rRNA gene from each DNA sample using the primers shown in online supplementary table S1. PCR was performed in triplicate on all DNA extracts using a MJ Research PTC-200P Thermal Cycler (MJ Research, Waltham, USA). PCR mixtures $(25 \mu \mathrm{l})$ contained $1 \times$ buffer $(20 \mathrm{mM}$ Tris/HCl, pH 8.4, $50 \mathrm{mM} \mathrm{KCl}), 1.5 \mathrm{mM}$ $\mathrm{MgCl}_{2}, 200 \mu \mathrm{M}$ each dNTP, $1.25 \mathrm{U}$ Taq polymerase (New England Biolabs, Hitchin, UK), 5 pmol each primer and $10 \mathrm{ng}$ DNA. The PCR conditions were: $95^{\circ} \mathrm{C}$ for $5 \mathrm{~min}$ initial denaturation, followed by 25 cycles of amplification at $95^{\circ} \mathrm{C}$ denaturation for $30 \mathrm{~s}$, annealing at $55^{\circ} \mathrm{C}$ for $40 \mathrm{~s}$, and extension of $72^{\circ} \mathrm{C}$ for $1 \mathrm{~min}$, with a final extension of $72^{\circ} \mathrm{C}$ for $5 \mathrm{~min}$. PCR products were pooled for each sample, purified using a Qiagen PCR purification kit, quantified and equimolar amounts pooled before running on a $1 / 4$ of a PTP (Pico titre plate) using titanium chemistry (AGOWA, Berlin, Germany). The sequences were binned according to their sample source and processed via RDP's pyropipeline 2728 to remove any reads that were less than $250 \mathrm{bp}$ and contained any ambiguities. The filtered sequences were classified using the RDP classifier and the relative proportions of phyla and families determined. The resulting microbial levels at phylum, class and family levels from each group (sham and RYGB) at postoperative weeks 2 and 8 were statistically compared using the Student t test.

\section{RESULTS AND DISCUSSION Effect of RYGB surgery on weight loss}

The multimodal effects of RYGB on the systemic metabolic and bacterial phenotype can be described in terms of the BRAVE effects (figure 1), ${ }^{4-11}$ which led to a decrease in postoperative body weight and food consumption (table 1) in a non-obese surgical rat model (to assess objectively the metabolic effects of surgery independently of any pre-existing metabolic dysfunction). These metabolic changes result in altered gastric and intestinal conditions, including a reduction of acid production with a resultant increase in $\mathrm{pH}^{29}$ and a suggested alteration in gut oxygen bioavailability, ${ }^{22}$ and exert a substantial effect not only on the host metabolism but also on gastrointestinal ecology, mainly manifested in a reduction in Firmicutes (includes the clostridial family) and Bacteroidetes, with a corresponding increase in Proteobacteria (Gammaproteobacteria family, figure 2). These changes are consistent with findings in a previous study of three human surgical subjects and six controls. ${ }^{22}$ The surgically altered anatomy, flow of nutrients and weight loss was associated with a global change in the urinary and faecal profiles reflecting (a) increased oligosaccharide fermentation in the gut, (b) biogenesis of $p$-cresol and related compounds, and (c) generation of amines, which may mechanistically contribute to body weight loss and metabolic enhancements. We summarise the effects of RYGB surgery in male Wistar rats $(n=14)$ over an 8week period after surgery in comparison with a sham-operated control group $(n=16)$ according to the BRAVE framework ${ }^{4}$ 9-11 and define the microbial-metabolic interactions that contribute to our current understanding of how RYGB surgery acts to reduce obesity and type 2 diabetes.

\section{Bile flow changes}

The gastric bypass procedure modifies the anatomical location at which bile enters the upper gastrointestinal tract via the biliopancreatic limb of the Roux-en-Y construction and increases primary and secondary serum bile acid concentrations, ${ }^{30}$ including taurochenodeoxycholic acid, taurodeoxycholic acid, glycocholic acid, glycochenodeoxycholic acid and glycodeoxycholic acid. ${ }^{31}$ A targeted ultra-performance liquid- 
Figure 1 Metabolic modulation following metabolic Roux-en-Y gastric bypass surgery (RYGB). The surgical diagram of our animal model of RYGB is effects (coloured box outlines) at relevant anatomical sites. Within each box we describe the physiological, biochemical and microbiological effects of surgery at each site (coloured text), and further describe whether these effects were noted in this study, derived from the literature, or our hypothesis based on experimental results and literature (numbered label key). GABA, g-aminobutyric acid; GLP-1, glucagonlike peptide-1; PYY, peptide YY; TCA: tricarboxylic acid. categorised according to the BRAVE

\section{The Roux-en-Y Gastric Bypass in Relation to Physiological and Microbial Activities}

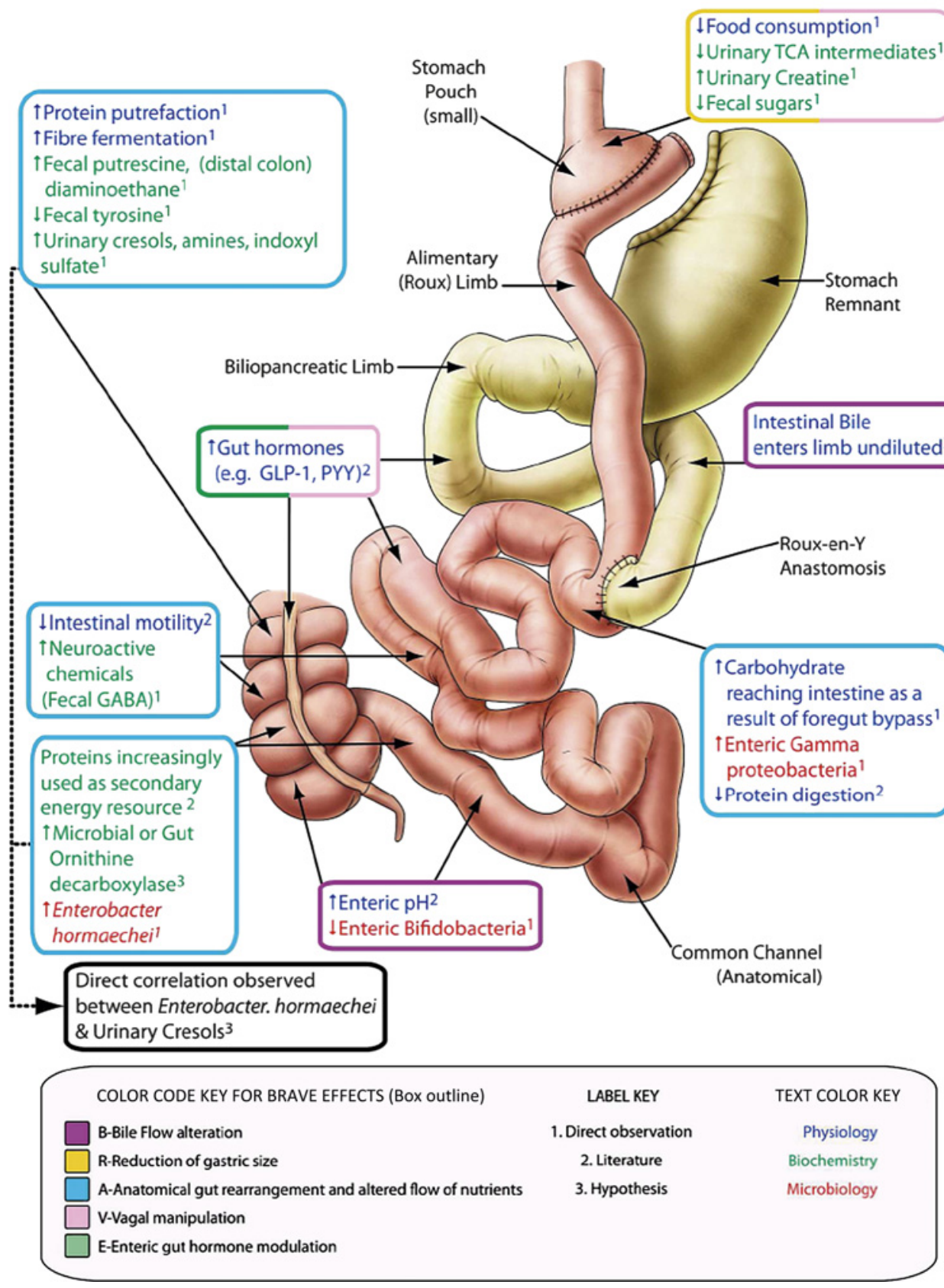

chromatography (UPLC)-MS method ${ }^{32}$ was used to profile the faecal bile acids in six selected animals in each group at three time points ( 1 week before and 6 and 8 weeks after the opera-

tion). We detected decreased faecal bile acids, mainly unconjugated, from 6 weeks following RYBG (online supplementary figure S1). The relative concentration of taurine-conjugated and

Table 1 Surgical outcomes, body weight and food consumption in the study population

\begin{tabular}{|c|c|c|c|c|c|c|c|c|c|c|c|c|c|c|}
\hline \multirow[b]{2}{*}{ Time points } & \multicolumn{2}{|c|}{ Number of rats } & \multicolumn{2}{|c|}{$\begin{array}{l}\text { Number for } \\
\text { NMR analysis }\end{array}$} & \multicolumn{2}{|c|}{$\begin{array}{l}\text { Number for } \\
\text { UPLC-MS } \\
\text { analysis } \\
\end{array}$} & \multicolumn{2}{|c|}{$\begin{array}{l}\text { Number for } \\
\text { faecal bacterial } \\
\text { analysis }\end{array}$} & \multicolumn{2}{|c|}{ Body weight (g) } & \multirow{2}{*}{$\begin{array}{l}\text { t-Test } \\
\text { p value }\end{array}$} & \multicolumn{2}{|c|}{$\begin{array}{l}\text { Food intake } \\
\text { RYBG (g) }\end{array}$} & \multirow{2}{*}{$\begin{array}{l}\mathrm{t} \text {-Test } \\
\text { p value } \\
\end{array}$} \\
\hline & Sham & Bypass & Sham & Bypass & Sham & Bypass & Sham & Bypass & Sham & Bypass & & Sham & Bypass & \\
\hline Week -1 (Pre-op) & 18 & 18 & 18 & 18 & 6 & 6 & 0 & 0 & $341.9 \pm 30.7$ & $346.6 \pm 35.8$ & 0.71 & $24.7 \pm 2.8$ & $25.7 \pm 2.7$ & 0.31 \\
\hline 2 (Post-op) & 16 & 15 & 16 & 15 & 0 & 0 & 6 & 6 & $358.6 \pm 22.6$ & $288.6 \pm 29.1$ & $<0.001$ & $29.5 \pm 2.8$ & $14.3 \pm 3.2$ & $<0.001$ \\
\hline 4 (Post-op) & 16 & 15 & 16 & 15 & & & 0 & 0 & $383.8 \pm 21.7$ & $275.6 \pm 25.4$ & $<0.001$ & $30.9 \pm 2.7$ & $22.4 \pm 3.1$ & $<0.001$ \\
\hline 6 (Post-op) & 16 & 14 & 16 & 14 & 6 & 6 & 0 & 0 & $400.3 \pm 22.8$ & $270.9 \pm 32.7$ & $<0.001$ & $25.7 \pm 3.2$ & $18.9 \pm 5.1$ & $<0.001$ \\
\hline 8 (Post-op) & 16 & 14 & 16 & 14 & 6 & 6 & 6 & 6 & $419.8 \pm 25.3$ & $259.1 \pm 36.1$ & $<0.001$ & $20.9 \pm 4.9$ & $14.6 \pm 6.5$ & 0.007 \\
\hline
\end{tabular}

NMR, nuclear magnetic resonance; UPLC-MS, ultra-performance liquid chromatography-tandem mass spectrometry; RYGB, Roux-en-Y gastric bypass. 
A

Heatmap at distance 0.10

B01

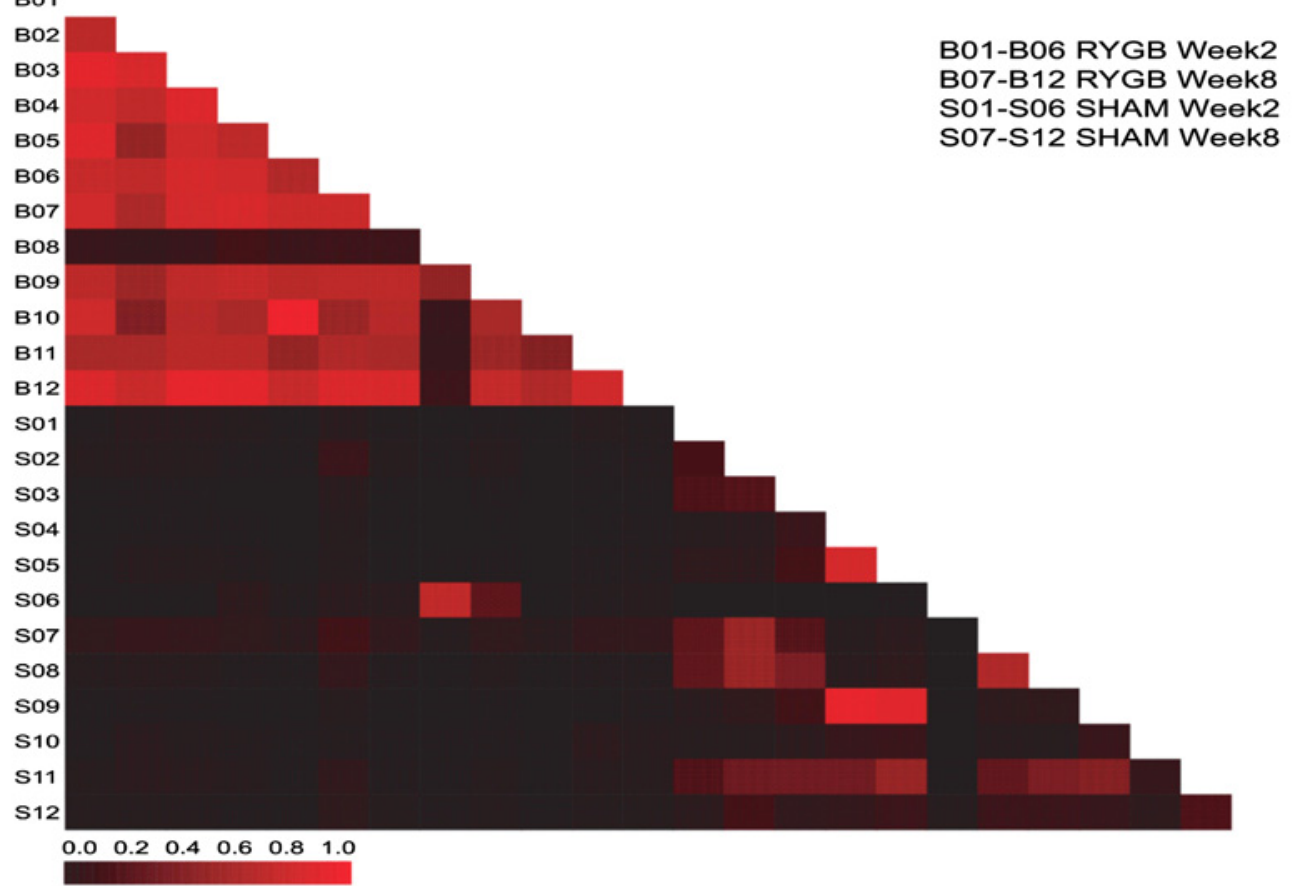

B01 B02 B03 B04 B05 B06 B07 B08 B09 B10 B11 B12 S01 S02 S03 S04 S05 S06 S07 S08 S09 S10 S11 S12

B01-B06 RYGB Week2

12 RYGB Week8

01-S06 SHAM Week2

B०

B

SHAM
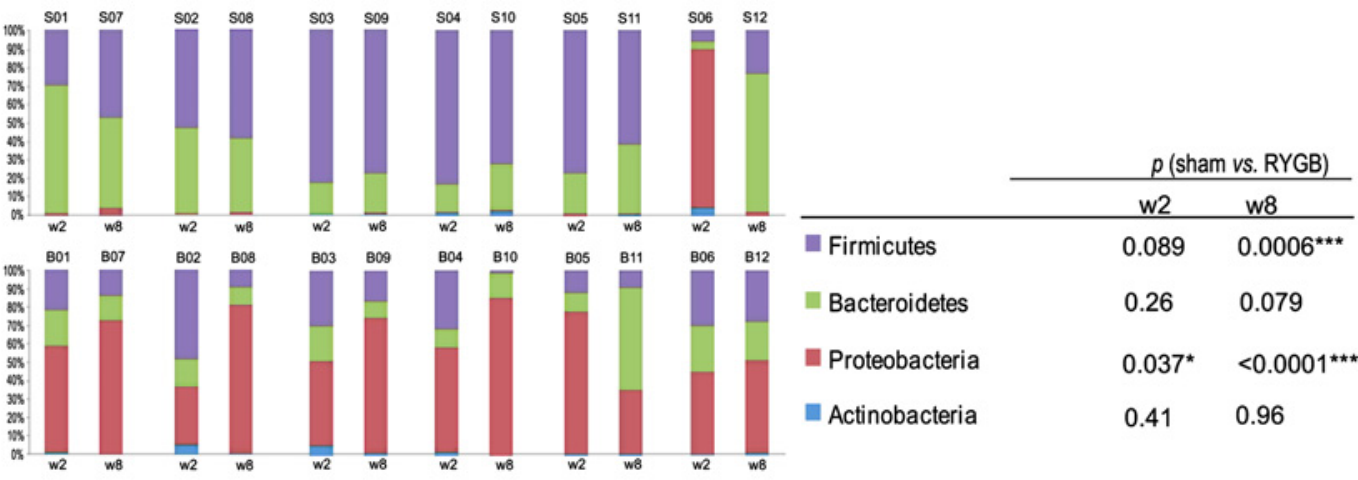

RYGB

Bacteroidetes

$0.26 \quad 0.079$

Proteobacteria $\quad 0.037^{\star}<0.0001^{\star \star \star}$

Actinobacteria $\quad 0.41 \quad 0.96$

C

SHAM

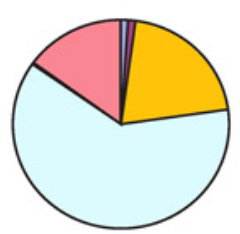

RYGB

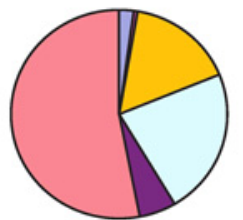

week 8
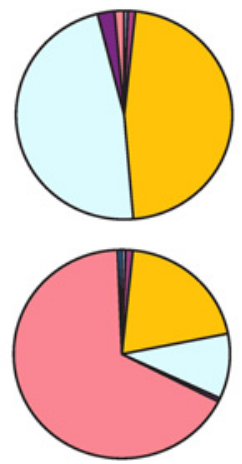

\begin{tabular}{|c|c|c|}
\hline & \multicolumn{2}{|c|}{$p$ (sham vs. RYGB) } \\
\hline & w2 & w8 \\
\hline Actinobacteria & 0.41 & 0.99 \\
\hline Bacilli & 0.41 & 0.90 \\
\hline Bacteroidetes & 0.47 & $0.03^{\star}$ \\
\hline Clostridia & $0.012^{* *}$ & $0.0048^{* * *}$ \\
\hline Erysipelotrichi & 0.11 & 0.39 \\
\hline Gammaproteobacteria & $0.036^{*}$ & $<0.0001^{\star \star \star}$ \\
\hline
\end{tabular}

Figure 2 A heat map (A) shows pairwise similarities between gut communities generated using MOTHUR with a cut-off of 0.10 . (B) Microbial composition of individual rat from sham control and Roux-en-Y gastric bypass surgery (RYGB)-operated groups at week 2 and 8 . The pie chart (C) shows the mean of each bacterial class level within the control $(N=6)$ and RYGB $(N=6)$ groups at week 2 and 8 . *The Student $t$ test was used to calculate the difference in each bacterial class between two groups for each time point. Of note was the anomalous behaviour of one of the sham rats (S06), which exhibited a high level of Proteobacteria at week 2, more consistent with the response of the RYGB-operated animals. This animal was reported to be unwell immediately after the sham surgical intervention, but subsequently recovered by week 8 after surgery, at which stage the microbial profile of this animal was similar to that of the other sham-operated animals. 
several unconjugated faecal bile acids in sham-operated rats was significantly higher than in the RYGB-operated animals at weeks 6 and 8 (taurine-conjugated: $15.6 \times, p=0.049$; unconjugated: $3.6 \times, p=0.026)$. This bile acid modulation may trigger the gut-brain-liver axis to achieve earlier satiety after meal consumption and improved glucose regulation. ${ }^{33}$

\section{Restriction of gastric size}

The dominant effect of RYGB surgery is to bypass the main body of the stomach and duodenum lower down the gastrointestinal tract so that nutrients are exposed to the small intestine (jejunum) immediately after transit through the oesophagus and a subtracted small gastric remnant (figure 1). In rats that underwent RYGB surgery ( $n=14$, mean preoperative weight $347 \mathrm{~g}$ ), we measured a mean \pm SD weight loss of $87.5 \pm 50.8 \mathrm{~g}$ over an 8-week post-surgical period, in contrast with a matched control group of sham-operated rats $(n=16$, mean preoperative weight $342 \mathrm{~g}$ ) which gained $77.9 \pm 39.7 \mathrm{~g}$, with a corresponding food consumption of $14.6 \mathrm{~g} /$ day for the RYGB group compared with $20.9 \mathrm{~g} /$ day for the sham group. These effects provide a degree of calorie restriction. Experimental caloric restriction has been associated with specific changes in the metabolic phenotype of experimental animals. We have previously demonstrated $^{34}$ that calorie restriction in rats results in a decrease in 2-oxoglutarate and an increase in creatine. Urinary metabolites (including succinate, 2-oxoglutarate, citrate and fumarate) of the RYGB-operated rats, measured by ${ }^{1} \mathrm{H}$ NMR spectroscopy at 2, 4, 6 and 8 weeks, were decreased at all observation time points (table 2). This observation, which persisted up to 8 weeks after the operation, indicates long-term modulation of cellular energy metabolism through increased utilisation of tricarboxylic acid (TCA) cycle intermediates. These findings are consistent with the results of calorie restriction where substrates are upregulated as a result of an acidotic effect, although we did not find a clear link between TCA cycle intermediates and animal body weight. These changes may therefore indicate renal tubular acidosis associated with an increased flux of TCA cycle intermediates in the mitochondrion ${ }^{35}$ and may contribute as a postoperative mechanism of weight loss and metabolic enhancement. Other studies of cellular energy metabolism after gastric bypass surgery have shown downregulation of mitochondrial complex I-IV after RYGB in diet-

Table 2 Summary of metabolic changes in urinary (u) and faecal (f) NMR profiles

\begin{tabular}{|c|c|c|c|c|c|}
\hline \multirow[b]{2}{*}{ Class } & \multirow[b]{2}{*}{ Metabolites } & \multicolumn{4}{|c|}{ 0-PLS-DA models of RYGB-operated and sham control rats } \\
\hline & & 2-week post-op & 4-week post-op & 6-week post-op & 8-week post-op \\
\hline \multirow[t]{4}{*}{ TCA cycle } & Succinate & $-0.6977(u)$ & $-0.6591(u) ;+0.5879(f)$ & $-0.8562(u)$ & $-0.7674(\mathrm{u}) ;+0.6286(\mathrm{f})$ \\
\hline & 2-0xoglutarate & $-0.6509(u)$ & $-0.6333(\mathrm{u}) ;{ }^{*}+0.6170(\mathrm{u})$ & $-0.8084(u)$ & $-0.8153(u)$ \\
\hline & Citrate & $-0.6090(u)$ & $-0.4856(u)$ & $-0.6150(u)$ & $-0.5045(u)$ \\
\hline & Fumarate & $-0.6518(u)$ & $-0.6374(u)$ & $-0.8703(u)$ & $-0.7200(u)$ \\
\hline \multirow[t]{5}{*}{ Amines } & Trimethylamine $N$-oxide & $+0.5183(u) ;{ }^{*}+0.7718(u)$ & $+0.5755(\mathrm{u}) ;{ }^{*}+0.6994(\mathrm{u})$ & $+0.6311(u) ; *+0.6241(u)$ & $+0.6937(u) ;{ }^{*}+0.7320(u)$ \\
\hline & Trimethylamine & $+0.6016(f)$ & $+0.8156(f)$ & & $+0.6865(f)$ \\
\hline & Methylamine & $+0.5196(f)$ & $+0.7819(f)$ & $+0.6933(f)$ & $+0.7167(\mathrm{f})$ \\
\hline & Putrescine & $+0.6133(f)$ & $+0.6601(f)$ & $+0.7048(f)$ & $+0.7328(f)$ \\
\hline & Diaminoethane & & $+0.5170(f)$ & $+0.6573(\mathrm{f})$ & $+0.7230(f)$ \\
\hline \multirow[t]{12}{*}{ Microbial activity } & Oligosaccharides & & & $-0.6144(f)$ & $-0.6461(f)$ \\
\hline & Acetate & $+0.5415(f)$ & & $+0.5413(f)$ & \\
\hline & Propionate & & $+0.5916(f)$ & $+0.5186(f)$ & \\
\hline & $p$-Cresol glucuronide & $+0.7785(u)$ & $+0.6304(\mathrm{u})$ & $+0.7116(u)$ & $+0.7225(\mathrm{u}) ; *+0.6367(\mathrm{u})$ \\
\hline & $p$-Cresol sulphate & $+0.7980(u)$ & $+0.6911(u)$ & $+0.6501(u)$ & $+0.7303(u)$ \\
\hline & 5-Aminovalerate & $+0.6977(u)$ & $+0.5518(u)$ & $+0.6297(u)$ & $+0.6921(u)$ \\
\hline & Phenylacetylglycine & $+0.9192(u) ; *+0.5416(u)$ & $+0.7038(u)$ & $+0.8432(u){ }^{*}+0.5497(u)$ & $+0.7533(u)$ \\
\hline & $p$-Hydroxyphenylacetate & $+0.7129(u)$ & $+0.5207(u)$ & $+0.6546(u)$ & $+0.6176(u)$ \\
\hline & Hippurate & & & $-0.8768(u)$ & $-0.6692(u)$ \\
\hline & Methanol & & & $+0.5561(f)$ & $+0.5167(f)$ \\
\hline & Formate & & & $+0.6820(f)$ & $+0.5907(f)$ \\
\hline & Tyrosine & $-0.6139(f)$ & $-0.6726(f)$ & $-0.6543(f) ; *+0.5820(f)$ & $-0.6065(f)$ \\
\hline \multirow{2}{*}{$\begin{array}{l}\text { Peripheral neuroactive } \\
\text { intermediate }\end{array}$} & $\gamma$-Amino-N-butyrate & $+0.7036(f)$ & $+0.7373(f)$ & $+0.7038(f)$ & $+0.6806(f)$ \\
\hline & Glutamate & & & $+0.5514(\mathrm{f})$ & $+0.5914(\mathrm{f})$ \\
\hline Urea cycle & Aspartate & $-0.6653(f)$ & $-0.7314(\mathrm{f})$ & $-0.6881(f)$ & $-0.6999(f)$ \\
\hline \multirow[t]{3}{*}{ Muscle metabolism } & Creatine & $+0.6619(u)$ & $+0.4473(u)$ & $+0.7781(u)$ & $+0.7294(u)$ \\
\hline & Creatinine & & & $*+0.6337(u)$ & $-0.7473(\mathrm{u}) i^{*}+0.6424(\mathrm{u})$ \\
\hline & Lactate & $+0.5871(\mathrm{f})$ & & & \\
\hline Renal metabolism & Indoxyl sulphate & $+0.8598(u)$ & $+0.7271(u)$ & $+0.6550(u) ;^{*}+0.7770(u)$ & $+0.6542(u)$ \\
\hline \multirow[t]{4}{*}{ Amino acids } & Valine & & & $+0.5822(\mathrm{f})$ & \\
\hline & Phenylalanine & & & $+0.5849(\mathrm{f}) ;{ }^{*}+0.5719(\mathrm{f})$ & \\
\hline & Alanine & & & ${ }^{*}+0.5636(f)$ & \\
\hline & Glycine & $+0.6577(f)$ & $+0.6317(\mathrm{f})$ & $+0.5698(f) ; *+0.6521(f)$ & $+0.5377(\mathrm{f})$ \\
\hline \multirow[t]{4}{*}{ Others } & N-Acetyl glycoproteins $2.06(s) \dagger$ & & & $-0.7523(\mathrm{f})$ & $-0.6160(f) ; *-0.5648(f)$ \\
\hline & Ethanol & & & $+0.6365(f)$ & $+0.6904(f)$ \\
\hline & Phenylalanine & & & $+0.5849(\mathrm{f}) ;{ }^{*}+0.5719(\mathrm{f})$ & \\
\hline & 2-0xoadipate & $-0.8260(u)$ & $-0.6238(\mathrm{u})$ & $-0.9030(u)$ & $-0.9045(u)$ \\
\hline
\end{tabular}

' + ' indicates a higher concentration of metabolites in RYGB-operated rats; ' - ' indicates a lower concentration of metabolites in RYGB-operated rats.

*Labelled metabolites are found to be changed in sham control rats in comparison with pre-operation, where ' $+1 /{ }^{\prime}$ ' indicates higher/lower concentrations of metabolites post-sham.

†Putative assignment.

The numbers are the coefficient values. NMR, nuclear magnetic resonance; RYGB, Roux-en-Y gastric bypass; TCA, tricarboxylic acid. 
induced obese Sprague-Dawley rats and postoperative human subjects, ${ }^{36}{ }^{37}$ which is also consistent with the findings of increased energy efficiency, calorie restriction, ${ }^{22}$ and the decrease in nutrient substrate noted after these operations ('I don't like burgers anymore syndrome'). ${ }^{10}$ Rhabdomyolysis (muscle lysis and metabolism) is a rare but recognised complication of gastric bypass surgery ${ }^{38}$ and is also observed in extreme calorie restriction. ${ }^{39}$ The criterion for rhabdomyolysis diagnosis is a more than fivefold increase in normal creatine kinase activity in the plasma. The higher urinary concentration of creatine in this study may therefore reflect increased muscle protein catabolism after surgery.

\section{Altered gastrointestinal anatomy}

The RYGB procedure results in a large stomach remnant (up to $1000 \mathrm{ml}$ in humans and $5 \mathrm{ml}$ in rats) with an associated segment of small bowel being bypassed lower down the upper gastrointestinal tract (figure 1). As a result, ingested nutrients are only exposed to a small stomach pouch $(\sim 15-30 \mathrm{ml}$ in man and $0.25 \mathrm{ml}$ in rats) before entering the small intestine earlier than the standard gastrointestinal configuration ${ }^{11}$ (figure 1). Consequently, the distal small intestine receives a higher load of nutrients that have not been exposed to the standard volume of stomach mucosa. RYGB profoundly disrupts the gut microbial ecology, as evidenced in the 454 sequencing data of six randomly selected animals from each group at 2 and 8 weeks after surgery. We show a dramatic increase (52-fold) in the relative proportion of phylum Proteobacteria (mainly the order Enterobacteriaceae), with a corresponding but less dramatic fall in the concentration of phylum Firmicutes (4.5-fold reduction-mainly the family Peptostreptococcaceae) in RYGB-operated rats (figure 2B). As the food intake at 2 and 8 weeks was similar, but there were pronounced changes in microbial composition between these postoperative time points, this may indicate that the surgical effect on gut microbiota was at least partially independent of food intake. The most striking alteration in microbial ecology after RYGB surgery was the growth of the class Gammaproteobacteria, particularly the species Enterobacter hormaechei (figures 2C and S2), which has not been reported previously. This is a Gram-negative bacterium with extended-spectrum $\beta$-lactamase activity which has previously been described as a nosocomial pathogen, although its role in gut and systemic metabolism requires further investigation. ${ }^{40}$ Selection for this bacterium may be derived from the effects of surgically altered gastrointestinal anatomy and inherent bacterial $\beta$-lactamase activity. The RYGB operation provides an anatomical pathway by which carbohydrates directly enter the ileum without prior exposure to the main body of the stomach. This favours the growth of enterobacterial species, as they display a high level of flexibility in fermenting carbohydrates. ${ }^{41}$ The faecal metabolite profile also showed evidence of altered energy metabolism, with the spectral region containing oligosaccharides and other sugars decreasing in relative percentage in the RYGB group, which is consistent with an altered microbial ecology favouring oligosaccharide use in the postoperative gut. ${ }^{42}$ Furthermore, the increased concentrations of various amines (methylamine, trimethylamine) in faecal extracts (table 2) reflects the fundamental role of the intestinal microbiota after RYGB surgery in the provision of methylamine and trimethylamine from the catabolism of precursors such as choline. $^{43}$

A further consequence of RYGB surgery is to modulate gastric emptying and intestinal mobility, which have been demonstrated to decrease after surgery such that $\sim 40 \%$ of human patients show very slow or no emptying in upper gastrointes- tinal contrast studies 1 year after surgery, ${ }^{44} 45$ and rodents show an $\sim 40 \%$ decrease in 30-min intestinal transit time after RYGB compared with controls. This is associated with the effects of proximal surgical vagotomy ${ }^{46}$ and the action of gut peptides such as ghrelin, GLP-1 and PYY on gastric and intestinal motility. ${ }^{47}$ Reduced upper-gastrointestinal motility lengthens intestinal exposure times, promoting favourable conditions for protein putrefaction. Consequently, incompletely digested proteins reaching the colon because of the surgical bypass of the foregut are likely to result in high bioavailability of proteins in the hindgut. As a result, several polyamines such as putrescine and diaminoethane (aliphatic biogenic amine) arise from microbial catabolic processes, ${ }^{48}$ as demonstrated by our faecal extract results. In our study, most faecal metabonomic changes had occurred by week 2 and persisted at post operation week 8 . The neuroactive peptide, $\gamma$-aminobutyric acid (GABA), is also increased in post-RYGB faecal samples and has also been shown to be derived from the microbial processing of putrescine. ${ }^{49}$ Increased expression of faecal GABA is consistent with the welldefined increase in GLP-1 after RYGB. ${ }^{12}$ GABA stimulates GLP-1 release from intestinal cells, ${ }^{50}$ and raised GLP-1 concentrations in turn promote GABA formation by pancreatic $\beta$ cells, with autocrine and paracrine effects, including $\alpha$ cell regulation to inhibit glucagon release, acinar cell activity to amplify cholecystokinin release, and immunomodulatory actions on infiltrating $T$ cells to suppress autoimmune damage. ${ }^{46}$ Increased brain GABA concentrations also reflect the hypothalamic GABAmediated control of food intake. ${ }^{5152}$

\section{Vagal nerve effects and enterohumoral (gut hormone) activity} Vagal nerve innervation and neurohormonal processes play a key role in the short-term regulation of food intake and the release of key gastrointestinal hormones including gastrin and secretin. ${ }^{12}$ Owing to the variation in vagal anatomy, operative surgical dissection near the stomach can lead to vagotomies of the gastric pouch and gastric remnant with varying results. ${ }^{53}$ Surgical vagal nerve stimulation can attenuate weight gain, ${ }^{54}$ and we have previously demonstrated that distal vagal-sparing bariatric procedures can enhance weight loss. ${ }^{55}$ Some of the weight loss effects of gastric bypass may reflect the surgical regulation of vagal activity in the gut-brain-liver axis in addition to the role of the vagus nerve in transmitting some of the appetite-suppression signals of modulated gut hormones after surgery. ${ }^{12}$ The direct effects of surgical vagal manipulation on the systemic metabolism are currently unclear; however, the increased awareness of vagal transection effects on appetite signalling ${ }^{56}$ may identify novel food-regulation mechanisms after surgery.

\section{Cross-talk between mammalian metabolism and the intestinal} microbiome and its effect on weight loss after RYGB operation The most striking metabolic feature of RYGB-operated rats is an increase in the diversity and complexity of signals in the aromatic region of the ${ }^{1} \mathrm{H}$ NMR urinary spectra (online supplementary figure S3), corresponding to increased concentrations of $p$-cresyl glucuronide, $p$-cresyl sulphate, 5-aminovalerate, phenylacetylglycine (PAG), p-hydroxyphenylacetate and indoxyl sulphate (figure $3 \mathrm{~A}, \mathrm{~B}$, table 2 ). The aromatic region of the ${ }^{1} \mathrm{H}$ NMR spectrum provides a convenient spectral window on gut microbial activity, ${ }^{57}$ and many of the metabolites with signals in the range $\delta 6.5-8.0$ are derived from mammalian-microbial co-metabolism. ${ }^{57}$

Fermentation is one of the many functions provided by gut microbes, where those of the proximal colon are mainly responsible for enhanced calorific recovery from otherwise 


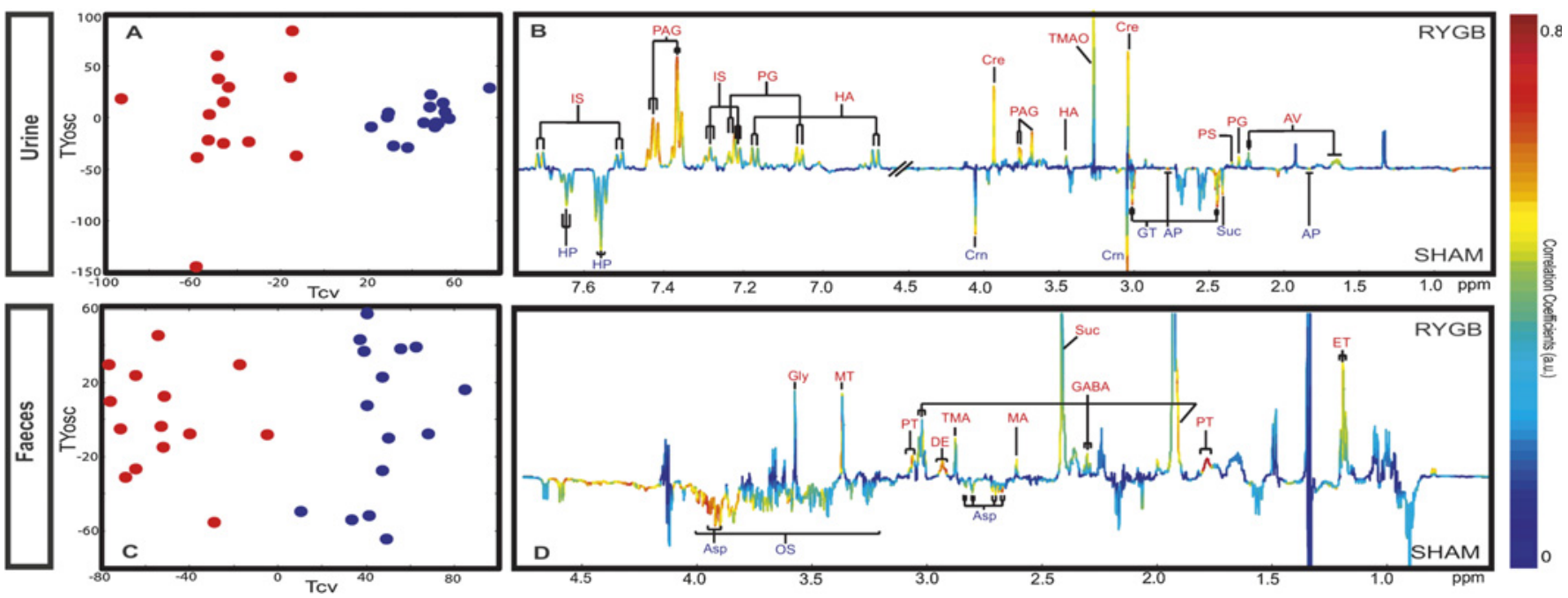

Figure 30 -PLS-DA cross-validation scores plots and coefficient plots of urinary $\left(A, B\left(R^{2} X=32 \% ; 0^{2} Y=0.86\right)\right)$ and faecal $\left(C, D,\left(R^{2} X=33.5 \%\right.\right.$; $\left.\left.\mathrm{O}^{2} Y=0.84\right)\right)$ nuclear magnetic resonance spectral data obtained from sham control (blue) and Roux-en-Y gastric bypass (RYGB)-operated rats (red) at week 8, reflecting the discrimination between these two groups. AP, 2-oxoadipate; Asp, aspartate; AV, 5-aminovalerate; Cre, creatine; Crn, creatinine; $\mathrm{DE}$, diaminoethane; ET, ethanol; FA, formate; FM, fumarate; GABA, $\gamma$-amino- $N$-butyrate; Gly, glycine; GT, 2-oxoglutarate; HA, $p$-hydroxyphenylacetate; HP, hippurate; IS, indoxyl sulphate; Lac, lactate; MA, methylamine; OS, oligosaccharides; PAG, phenylacetylglycine; PG, $p$-cresyl glucuronide; PS, p-cresyl sulphate; PT, putrescine; Suc, succinate; TMA, trimethylamine; TMA0, trimethylamine $N$-oxide; Ura, uracil.

indigestible polysaccharides. ${ }^{58}$ The lower concentrations of oligosaccharides and higher concentrations of short-chain fatty acids (table 2), which are major products of fermentation, suggest increased microbial fermentation activity. There was a positive trend between fermentation-generated compounds and lower body weight, although this was not significant, but may suggest a role in metabolic modulation and metabolic syndrome resolution after surgery. ${ }^{3} 9$ The efficiency of microbially mediated energy recovery is determined by the composition of the microbiome.

To probe the deeper association between the changes in bacterial and metabolic phenotypes we correlated key RYBGdiscriminatory bacterial classes, and selected species, directly with the metabolic profiles. A significant proportional increase in the Gammaproteobacteria in RYGB-operated rats correlated positively with urinary $p$-hydroxyphenylacetate, $p$-cresyl glucuronide, $p$-cresyl sulphate, creatine, PAG and indoxyl sulphate and with faecal succinate, putrescine, diaminoethane, uracil, glycine, methylamine and formate (figure 4). Clostridia showed an association with decreased urinary $p$-cresyl glucuronide, PAG and creatine, decreased faecal succinate, glycine, uracil and formate, and increased faecal oligosaccharides (figure 4). The main contributor to the increase in Gammaproteobacteria was E. hormaechei, which manifested a 200-fold and 42.8-fold increase at weeks 2 and 8 after RYGB surgery, respectively. In the case of E. hormaechei, strong direct covariation with p-cresyl derivatives,
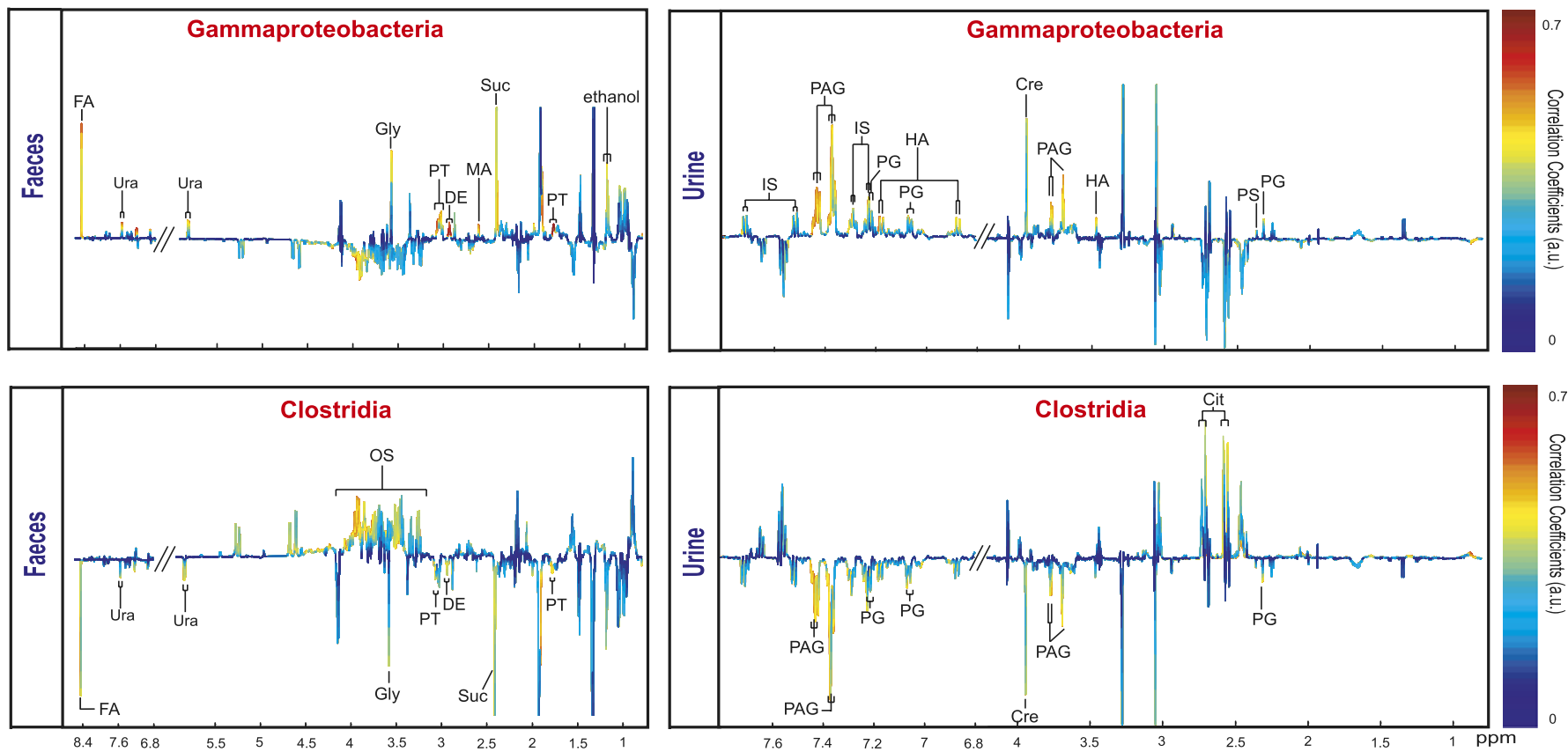

Figure 4 O-PLS regression loadings plot showing correlation between the combination of urinary and faecal nuclear magnetic resonance spectral data and Gammaproteobacteria $\left(Q^{2} Y=0.58 ; R^{2} X=18.0 \%\right)$ and Clostridia $\left(Q^{2} Y=0.45 ; R^{2} X=18.7 \%\right)$ levels. 
p-cresyl glucuronide and p-cresyl sulphate, PAG and creatine was detected (online supplementary figure S5). Thus, although it has been reported that some species of Clostridia produce phenol and $p$-cresol together with ammonia and hydrogen by anaerobic degradation of aromatic amino acids such as tyrosine, ${ }^{59}$ our findings suggest that Gammaproteobacteria (predominantly in this case E. hormaechei) may also contribute to the pool of cresol metabolites in the urine. This finding is consistent with previous reports that changes in gut Enterobacter spp. are associated with corresponding alterations in $p$-cresol metabolite concentrations. ${ }^{60}$

Urinary concentrations of PAG, $p$-cresyl glucuronide, 5 -aminovalerate, $p$-cresyl sulphate, creatine and $p$-hydroxyphenylacetate and faecal concentrations of uracil, putrescine and methylamine increased as body weight decreased (online supplementary figure S6A). High levels of Enterobacteriaceae and Pasteurellaceae are associated with weight loss, whereas Lachinospiraceae, Incertae Sedis XIII and Prevotellaceae increased with weight gain (online supplementary figure S6B). Although the correlation between body weight and Lactobacillaceae is not strong, this may be due to the dichotomous response whereby RYGB-operated animals have very low concentrations of Lactobacillaceae, whereas almost all of the sham-operated animals exhibited higher concentrations. Enterobacteriaceae concentrations exhibit a strong correlation with surgical weight loss and urinary PAG, putrescine, uracil, $p$-cresyl glucuronide, creatine and methylamine concentrations, whereas urinary excretion of p-cresyl sulphate correlates with Bifidobacteriaceae and Micrococcaceae (online supplementary figure S7).

\section{Does bariatric surgery have a short-term gain and a long-term loss?}

Bariatric surgery is currently the most effective long-term treatment for morbid obesity. It is associated with decreased cardiovascular risk and cancer incidence compared with obese controls. $^{3} 48$ Bariatric surgery-induced bacterial and metabolic alterations can contribute to surgical weight loss, beneficial metabolic outcomes, and decreased mortality. However, a shift towards the Gammaproteobacteria as a major component of the microbiota and increase in $p$-cresol derivatives and another uraemic toxin, indoxyl sulphate, could have long-term effects on host health, with unpredictable outcomes. Recent evidence also identifies the bacterium E. hormaechei as an apoptosis-inducing agent in human epithelial cells. ${ }^{61}$ A deeper understanding of the disease-modifying mechanisms of bariatric surgery is therefore essential. Studying such metabolic interactions through a topdown systems biology approach can thus improve our current understanding of surgery to provide improved outcomes and novel treatments for obesity and metabolic disorders.

At 8 weeks after RYGB, urinary creatinine concentrations were lower and may reflect improved postoperative creatinine clearance or surgically induced kidney or muscle injury, as the uraemic toxin, indoxyl sulphate, was also found to increase in the RYGB-operated group. The role of these metabolic changes in kidney function requires further investigation, as clinical studies have shown the beneficial effects of RYGB ${ }^{62}$ and GLP- $1^{63}$ on renal function.

The present work represents a systematic and dynamic investigation of the metabolic and bacterial effects of the RYGB procedure in Wistar rats using bacterial profiling and NMRbased metabonomic approaches. Both longitudinal (time effect) and horizontal (surgery effect) results are prominent in metabolic and bacterial signatures, where we also provide triangular (body weight loss, metabolite profiles and bacterial compositions) correlations. We uncover mechanistic insights into the role of RYGB in achieving weight loss and metabolic enhancement. This includes the surgical effect of modulating biochemical cross-talk between the gut microbiome and systemic host metabolism. Here we identify E. hormaechei as a long-term key contributor to gut ecology after surgery. This organism is an excellent $\beta$-lactamase producer ${ }^{40}$ and therefore may be resistant to preoperative antibiotics (amoxycillin and flucloxacillin, both $\beta$-lactams), thus enhancing its opportunity to colonise soon after the operation; however, interestingly in the RYGB group, bacterial levels stayed high, whereas this was not the case in sham-operated animals. These changes are associated with our findings of increased protein putrefaction after surgery. Furthermore, we support the increased awareness that these operations enhance weight loss through metabolic regulation of the gut-liver-brain axis and enhancement of mitochondrial energetic efficiency. These multisystem effects of surgical intervention suggest a profound contribution of integrated host and microbial metabolism underlying the sophisticated mechanisms behind the metabolic benefits of bariatric surgery.

Acknowledgements We thank Dr E Want for help in performing UPLC experiments and data analysis. We also thank Catherine Sulzmann for her artwork.

Funding This study received financial support from the Imperial College London Junior research fellowship to JVL and from the Wellcome Trust Research Training Fellowship to HA. We are grateful for support from the NIHR Biomedical Research Centre Funding Scheme.

\section{Competing interests None}

Ethics approval All animal experiments were approved by Charing Cross Research Ethics Committee, London, UK.

Contributors $\mathrm{HA}$ and TA designed research; $\mathrm{HA}$ and MB performed anima experiments and clinical measurement; JVL performed NMR analyses; JRM and JVL performed microbiomic and bioinformatic analyses; JVL, JRM, HA, CS, JKN, CWIR, SRB, AD, TA and EH analysed and interpreted data; JVL, JRM, HA, JK, JKN, TA and EH wrote the paper.

Provenance and peer review Not commissioned; externally peer reviewed.

\section{REFERENCES}

1. World Health Organization. Obesity and Overweight_Fact Sheet N³11. Geneva: World Health Organization, 2006.

2. International Diabetes Federation. Diabetes Atlas-Prevalence and Projections. Brussels, Belgium: International Diabetes Federation, 2008.

3. Ashrafian H, le Roux CW, Darzi A, et al. Effects of bariatric surgery on cardiovascular function. Circulation 2008;118:2091-102.

4. Ashrafian H, Athanasiou T, le Roux CW. Heart remodelling and obesity: the complexities and variation of cardiac geometry. Heart 2011;97:171-2.

5. Ashrafian H, Ahmed K, Rowland SP, et al. Metabolic surgery and cancer: protective effects of bariatric procedures. Cancer 2011;17:1788-99.

6. Ashrafian H, Darzi A, Athanasiou A. Bariatric surgery: can we afford to do it or deny doing it? Frontline Gastroenterol 2011;2:82-89.

7. Puterbaugh JS. The emperor's tailors: the failure of the medical weight loss paradigm and its causal role in the obesity of America. Diabetes Obes Metab 2009;11:557-70.

8. Sjostrom L, Narbro K, Sjostrom CD, et al. Effects of bariatric surgery on mortality in Swedish obese subjects. N Engl J Med 2007;357:741-52.

9. Ashrafian H, Athanasiou T, Li JV, et al. Diabetes resolution and hyperinsulinaemia after metabolic Roux-en-Y gastric bypass. Obes Rev 2011;12:e257-72.

10. Ashrafian H, Bueter M, Ahmed K, et al. Metabolic surgery: an evolution through bariatric animal models. Obes Rev 2010;11:907-20.

11. Ashrafian H, Darzi A, Athanasiou T. Autobionics: a new paradigm in regenerative medicine and surgery. Regen Med 2010;5:279-88.

12. Ashrafian H, le Roux CW. Metabolic surgery and gut hormones-a review of bariatric entero-humoral modulation. Physiol Behav 2009;97:620-31.

13. Clayton TA, Lindon JC, Cloarec 0, et al. Pharmaco-metabonomic phenotyping and personalized drug treatment. Nature 2006;440:1073-7.

14. Nicholson JK, Lindon JC. Systems biology: metabonomics. Nature 2008;455:1054-6.

15. Holmes E, Loo RL, Stamler J, et al. Human metabolic phenotype diversity and its association with diet and blood pressure. Nature 2008;453:396-400.

16. Holmes E, Wilson ID, Nicholson JK. Metabolic phenotyping in health and disease. Cell 2008;134:714-17. 
17. Waldram A, Holmes $E$, Wang $Y$, et al. Top-down systems biology modeling of host metabotype-microbiome associations in obese rodents. J Proteome Res 2009;8:2361-75

18. Williams R, Lenz EM, Wilson AJ, et al. A multi-analytical platform approach to the metabonomic analysis of plasma from normal and Zucker (fa/fa) obese rats. Mol Biosyst 2006;2:174-83

19. Ley RE, Turnbaugh PJ, Klein S, et al. Microbial ecology: human gut microbes associated with obesity. Nature 2006;444:1022-3.

20. Turnbaugh PJ, Hamady M, Yatsunenko $T$, et al. A core gut microbiome in obese and lean twins. Nature 2009;457:480-4.

21. Duncan SH, Lobley GE, Holtrop G, et al. Human colonic microbiota associated with diet, obesity and weight loss. Int J Obes (Lond) 2008;32:1720-4.

22. Zhang H, DiBaise JK, Zuccolo A, et al. Human gut microbiota in obesity and after gastric bypass. Proc Natl Acad Sci U S A 2009;106:2365-70.

23. Furet JP, Kong LC, Tap J, et al. Differential adaptation of human gut microbiota to bariatric surgery-induced weight loss: links with metabolic and low-grade inflammation markers. Diabetes 2010;59:3049-57.

24. Mutch DM, Fuhrmann JC, Rein D, et al. Metabolite profiling identifies candidate markers reflecting the clinical adaptations associated with Roux-en-Y gastric bypass surgery. PLoS One 2009:4:e7905.

25. Beckonert 0, Keun HC, Ebbels TM, et al. Metabolic profiling, metabolomic and metabonomic procedures for NMR spectroscopy of urine, plasma, serum and tissue extracts. Nat Protoc 2007:2:2692-703.

26. Nicholson JK, Foxall PJ, Spraul M, et al. $750 \mathrm{MHz} 1 \mathrm{H}$ and $1 \mathrm{H}-13 \mathrm{C}$ NMR spectroscopy of human blood plasma. Anal Chem 1995;67:793-811.

27. Cole JR, Wang Q, Cardenas E, et al. The Ribosomal Database Project: improved alignments and new tools for rRNA analysis. Nucleic Acids Res 2009;37: D141-5.

28. Schloss PD, Westcott SL, Ryabin T, et al. Introducing mothur: open-source, platform-independent, community-supported software for describing and comparing microbial communities. Appl Environ Microbiol 2009:75:7537-41.

29. Melissas J, Kampitakis E, Schoretsanitis G, et al. Does reduction in gastric acid secretion in bariatric surgery increase diet-induced thermogenesis? Obes Surg 2002:12:236-40.

30. Nakatani H, Kasama K, Oshiro T, et al. Serum bile acid along with plasma incretins and serum high-molecular weight adiponectin levels are increased after bariatric surgery. Metabolism 2009;58:1400-7.

31. Patti ME, Houten SM, Bianco AC, et al. Serum bile acids are higher in humans with prior gastric bypass: potential contribution to improved glucose and lipid metabolism. Obesity (Silver Spring) 2009;17:1671-7.

32. Want EJ, Coen M, Masson P, et al. Ultra performance liquid chromatography-mass spectrometry profiling of bile acid metabolites in biofluids: application to experimental toxicology studies. Anal Chem 2010;82:5282-9.

33. Wang PY, Caspi L, Lam CK, et al. Upper intestinal lipids trigger a gut-brain-liver axis to regulate glucose production. Nature 2008:452:1012-16.

34. Veselkov KA, Lindon JC, Ebbels TM, et al. Recursive segment-wise peak alignment of biological (1)h NMR spectra for improved metabolic biomarker recovery. Anal Chem 2009;81:56-66.

35. Nissim I, Yudkoff M. Adaptation of renal tricarboxylic acid cycle metabolism to various acid-base states: study with [3-13C,5-15N]glutamine. Miner Electrolyte Metab 1991;17:21-31.

36. Guijarro A, Osei-Hyiaman D, Harvey-White J, et al. Sustained weight loss after Roux-en-Y gastric bypass is characterized by down regulation of endocannabinoids and mitochondrial function. Ann Surg 2008;247:779-90.

37. Li J, Feuers RJ, Desai VG, et al. Surgical caloric restriction ameliorates mitochondrial electron transport dysfunction in obese females. Obes Surg 2007:17:800-8.

38. Koffman BM, Greenfield LJ, Ali II, et al. Neurologic complications after surgery for obesity. Muscle Nerve 2006:33:166-76.

39. Worley G, Claerhout SJ, Combs SP. Hypophosphatemia in malnourished children during refeeding. Clin Pediatr (Phila) 1998;37:347-52.

40. Townsend SM, Hurrell E, Caubilla-Barron J, et al. Characterization of an extendedspectrum beta-lactamase Enterobacter hormaechei nosocomial outbreak, and other
Enterobacter hormaechei misidentified as Cronobacter (Enterobacter) sakazakii. Microbiology 2008;154:3659-67

41. Le Bouguenec C, Schouler C. Sugar metabolism, an additional virulence factor in enterobacteria. Int J Med Microbiol 2011:301:1-6.

42. Claus SP, Tsang TM, Wang Y, et al. Systemic multicompartmental effects of the gut microbiome on mouse metabolic phenotypes. Mol Syst Biol 2008:4:219.

43. al-Waiz M, Mikov M, Mitchell SC, et al. The exogenous origin of trimethylamine in the mouse. Metabolism 1992;41:135-6.

44. Akkary E, Sidani S, Boonsiri J, et al. The paradox of the pouch: prompt emptying predicts improved weight loss after laparoscopic Roux-Y gastric bypass. Surg Endosc 2009;23:790-4.

45. Horowitz M, Collins PJ, Harding PE, et al. Gastric emptying after gastric bypass. Int J Obes 1986;10:117-21.

46. Urbain JL, Penninckx F, Siegel JA, et al. Effect of proximal vagotomy and Roux-en-Y diversion on gastric emptying kinetics in asymptomatic patients. Clin Nucl Med 1990;15:688-91.

47. Suzuki S, Ramos EJ, Goncalves CG, et al. Changes in Gl hormones and their effect on gastric emptying and transit times after Roux-en-Y gastric bypass in rat model. Surgery 2005;138:283-90

48. Welters CF, Dejong CH, Deutz NE, et al. Effects of parenteral arginine supplementation on the intestinal adaptive response after massive small bowel resection in the rat. J Surg Res 1999;85:259-66.

49. Kurihara S, Kato K, Asada K, et al. A putrescine-inducible pathway comprising PuuE-Ynel in which gamma-aminobutyrate is degraded into succinate in Escherichia coli K-12. J Bacteriol 2010;192:4582-91.

50. Gameiro A, Reimann F, Habib AM, et al. The neurotransmitters glycine and GABA stimulate glucagon-like peptide-1 release from the GLUTag cell line. J Physiol 2005:569:761-72

51. Nobrega JN, Snow J, Dixon LM, et al. Graded increases in brain GABA: differentia effects on feeding and other behaviours in rats. Behav Brain Res 1988;31:135-47.

52. Turenius $\mathbf{C I}$, Charles JR, Tsai DH, et al. The tuberal lateral hypothalamus is a majo target for GABAA-but not GABAB-mediated control of food intake. Brain Res 2009; 1283:65-72.

53. Sapala JA, Wood MH, Schuhknecht MP. Vagotomy at the time of gastric bypass: can it be harmful? Obes Surg 2004;14:575-6.

54. Sobocki J, Fourtanier G, Estany J, et al. Does vagal nerve stimulation affect body composition and metabolism? Experimental study of a new potential technique in bariatric surgery. Surgery 2006;139:209-16.

55. Bueter M, Lowenstein C, Ashrafian $\mathrm{H}$, et al. Vagal sparing surgical technique but not stoma size affects body weight loss in rodent model of gastric bypass. Obes Surg 2010;20:616-22.

56. Sundbom M, Holdstock C, Engstrom BE, et al. Early changes in ghrelin following Roux-en-Y gastric bypass: influence of vagal nerve functionality? Obes Surg 2007:17:304-10

57. Nicholson JK, Connelly J, Lindon JC, et al. Metabonomics: a platform for studying drug toxicity and gene function. Nat Rev Drug Discov 2002;1:153-61.

58. Nicholson JK, Holmes E, Wilson ID. Gut microorganisms, mammalian metabolism and personalized health care. Nat Rev Microbiol 2005;3:431-8.

59. Elsden SR, Hilton MG, Waller JM. The end products of the metabolism of aromatic amino acids by Clostridia. Arch Microbiol 1976;107:283-8.

60. Hida M, Aiba Y, Sawamura S, et al. Inhibition of the accumulation of uremic toxins in the blood and their precursors in the feces after oral administration of Lebenin, a lactic acid bacteria preparation, to uremic patients undergoing hemodialysis. Nephron 1996;74:349-55

61. Krzyminska S, Koczura R, Mokracka J, et al. Isolates of the Enterobacter cloacae complex induce apoptosis of human intestinal epithelial cells. Microb Pathog 2010;49:83-9.

62. Serpa Neto A, Bianco Rossi FM, Dal Moro Amarante R, et al. Effect of weight loss after Roux-en-Y gastric bypass, on renal function and blood pressure in morbidly obese patients. J Nephrol 2009;22:637-46.

63. Bueter M, Ahmed A, Ashrafian $\mathrm{H}$, et al. Bariatric surgery and hypertension. Surg Obes Relat Dis 2009;5:615-20. 